Emergency Ambulance Dispatch and Drive Times: An Analysis of Prehospital Vehicular Response in the Kingdom of Bhutan

Dr. Jenevieve Kincaid ${ }^{1}$, Dr. Charles Mize ${ }^{2}$, Mila Dorji ${ }^{3}$

1. University of Nevada Reno School of Medicine, Reno, United States

2. Bear/Badger Expeditionary and Retrieval Medicine, New Haven, United States

3. Yale University, New Haven, United States

Introduction: The Kingdom of Bhutan is a small, mountainous country with limited financial resources. Its population is scattered in hard-to-reach villages with poor road access. Ambulance drivers piloting Toyota Landcruisers provide the majority of the country's emergency response and are dispatched by the national emergency response center (Health Help Service/112) to calls in the nation's twenty districts.

Aim: By collecting and analyzing prehospital response data, we aimed to describe Bhutanese emergency medical response (EMS) ambulance activities and make system-wide recommendations to improve the speed of emergency vehicle dispatch, reduce the time between ambulance activation and ambulance arrival on scene, and adequately describe emergency vehicle drive time as it relates to distance driven.

Methods: The following data was compiled in Excel: Dispatch center phone records, EMS ambulance activation times, drive times, vehicle geospatial data, and written records of ambulance drivers. No identifiable data was collected.

Inclusion Criteria: All prehospital calls from 2017 and 2018 where complete data was available.

Exclusion Criteria: Complete data unavailable, i.e. geographic data without a matching call or report.

Statistical Tools: SPSS Statistics Version 25, NVivo 1212.2.0.3262.

Results: Preliminary analysis of the data shows a significant difference between data collected and data previously reported, the speed of emergency vehicular response and dispatch, drive times, and distance traveled. Facility transfer rather than scene response was found to take more time.

Discussion: Due to adverse road conditions, lengthy drive times, and an inadequate number of personnel and satellite ambulance locations, we recommend optimizing ambulance location using an optimization model that will minimize the number of ambulances needed and maximize response time. Future considerations may include adding a ground arm to the Bhutan Emergency Aeromedical Retrieval team, or a second aeromedical team in the eastern part of the country.

Prehosp Disaster Med 2019;34(Suppl. 1):s125

doi:10.1017/S1049023X19002693

\section{An Emergency and Disaster Course on Responding to Community and Family Healthcare Problems with Interprofessional Education for Undergraduate Medical, Nursing, and Dietitian Students \\ Miss Madelina Ariani ${ }^{1}$, Mrs. Bella Donna ${ }^{1}, M r$. Sutono Sutono ${ }^{2}$, Mrs. Yayuk Hartriyanti ${ }^{3}$, Mrs. Fitriana Fitriana ${ }^{4}$, Mr. Nandyan Wilasto ${ }^{5}$, Mrs. Gandes Rabayu ${ }^{6}$}

1. Center for Health Policy and Management, Faculty of Medicine, Public Health, and Nursing, Universitas Gadjah Mada, Yogyakarta, Indonesia

2. Program Study of Nursing, Faculty of Medicine, Public Health, and Nursing, Universitas Gadjah Mada, Yogyakarta, Indonesia

3. Health Nutrition Program Study, Faculty of Medicine, Public Health, and Nursing, Universitas Gadjah Mada, Yogyakarta, Indonesia

4. Community and Family Medicine, Faculty of Medicine, Public Health, and Nursing, Universitas Gadjah Mada, Yogyakarta, Indonesia

5. Health Policy Management Department, Faculty of Medicine, Public Health, and Nursing, Universitas Gadjah Mada, Yogyakarta, Indonesia

6. Academic Vice Dean, Faculty of Medicine, Public Health, and Nursing, Universitas Gadjah Mada, Yogyakarta, Indonesia

Introduction: Emergencies and disasters need inter-discipline and inter-professional approaches because many problems in a disaster are due to poor coordination and collaboration. The disaster events during a decade in Indonesia highlighted the limitations of the healthcare system in responding to large-scale public health problems. Disaster health preparedness is the key to an effective response to any problems in community and family. Thus, education for health students has become a priority. Aim: Preparing fourth-year health students to be aware of disaster health problems in family and community with an interprofessional approach.

Methods: Faculty of Medicine, Public Health, and Nursing UGM were prepared for the fourth year undergraduate health students through a semester "Emergency and Disaster Course" under Community Family and Healthcare with the Inter-professional Education Program, first given in 2016 for four hundred students. Mix method between class lecture, training skill, and simulation. The course goals are to (1) educate students on disaster health management, (2) understand the health preparedness and disaster family kit, and (3) define the principle of health worker's role and collaboration in disaster.

Results: The course was well received and at the 2017-2018 session was improved based on students and faculty feedback. Disaster knowledge of students changed. However, they still had a problem in communication between professions. And addition, they became aware of the function and each role of health profession competency during a disaster.

Discussion: A course for fourth-year health students about emergency and disaster health management is extremely relevant because they will be health workers soon. They must have good awareness, knowledge, and attitude to cope with disaster health problems in the future.

Prehosp Disaster Med 2019;34(Suppl. 1):s125

doi:10.1017/S1049023X1900270X

An Emergency Medical Triage Tool for Swiftwater Rescue Dr. David Jaslow ${ }^{1}$, Mr. Nathaniel Zahustecher ${ }^{1}$, Mr. Fred Ellinger ${ }^{1}, M r$. Derek Zecher ${ }^{1}$, Dr. Ryan Overberger ${ }^{2}$

1. Bryn Athyn Fire Company, Bryn Athyn, United States

2. Einstein Medical Center, Philadelphia, United States 